\title{
NECESIDAD DE TRATAMIENTO ENDODÓNCICO Y PREVALENCIA DE CARIES EN ESCOLARES DE 12 AÑOS EN LA PARROQUIA YANUNCAY CUENCA-ECUADOR 2016
}

\section{NEED FOR ENDODONTIC AND PREVALENCE TREATMENT OF CARIES 12 YEARS OLD SCHOOLSCHILDREN IN THE NEIGHBORHOOD YANUNCAY IN CUENCA-ECUADOR 2016}

\author{
Herrera Dayana. ${ }^{1 *}$, Apaza Frank. ${ }^{2}$,Pariona María del Carmen. ${ }^{3}$, Vilca Laura. ${ }^{4}$ \\ ${ }^{1}$ Universidad Católica de Cuenca.Ecuador. \\ 2 Od. Esp. y Est. de prégrado de la Universidad Andina Néstor Cáceres Velasquez.Perú. \\ ${ }^{3}$ Docente de la Universidad Católica de Cuenca.Ecuador. \\ 4 Est. maestría de Salud Pública Universidad Andina Néstor Cáceres Velasquez.Perú. \\ *dcherreraa92@est.ucacue.edu.ec
}

\begin{abstract}
Resumen
OBJETIVO: Determinar la frecuencia de necesidad de tratamiento endodóncico y la prevalencia de caries en escolares de 12 años en la Parroquia Yanuncay de la ciudad de Cuenca - Ecuador 2016. MATERIALES Y MÉTODOS: Estudio transversal de evaluación clínica bucal en 130 escolares de 12 años en la Parroquia Yanuncay de la ciudad de Cuenca - Ecuador 2016, esta muestra se calculó mediante fórmula en base a una población de 1398 escolares, se incluyeron pacientes con dentición permanente, fueron excluidos los que presentaron enfermedades sistémicas y malformaciones bucales, se utilizaron los criterios del índice de Pulpitis, Ulceras, Fístulas y Abscesos (P.U.F.A) para la necesidad de tratamiento endodóncico y los criterios epidemiológicos de la Organización Mundial de la Salud (OMS) para la prevalencia de caries. Los observadores fueron calibrados por un especialista, los padres firmaron el consentimiento y los escolares dieron el asentimiento informado. Todos los exámenes bucales fueron desarrollados con luz natural y espejo bucal, previo a la hora de su refrigerio. RESULTADOS: La muestra presentó $50 \%$ de escolares de cada sexo; $7 \%$ del total de la muestra presentaron necesidad de tratamiento endodóncico, principalmente por pulpitis; así mismo del total de escolares el $5 \%$ requiere tratamiento en una sola pieza dental y el $95 \%$ en 2 o más piezas dentales. El $39 \%$ de la muestra presentó caries. No existe diferencia significativa en la frecuencia de necesidad de tratamiento endodóncico ni en la prevalencia de caries entre ambos sexos $\left(\mathrm{X}^{2} \mathrm{p}>0.05\right)$
\end{abstract}

Palabras clave: Endodoncia, prevalencia, caries dental, pulpitis.

\begin{abstract}
AIM: To determine the frequency for the need for Endodontic treatment and the prevalence of cavities in 12 year old children in Yanuncay Cuenca - Ecuador 2016. MATERIALS AND METHODS: Cross-sectional study of oral clinical evaluation in 13012 year old children in the urban community in Yanuncay Cuenca-Ecuador, this sample was calculated using a formula based on a population of 1398 school children, including patients with permanent dentition, those who presented systemic diseases oral malformations were excluded, we used of P.U.F.A Index criteria for the need of endodontic treatment and the epidemiological criteria of the OMS for the prevalence of cavities. The observers were calibrated by a specialist, parents signed the consent form and school children gave the informed consent. All oral exams were developed under natural light and oral mirrors, previous to lunch time. RESULTS: The sample presented 50\% of each gender of the schoolchildren; $7 \%$ had the need for endodontic treatment, mainly by pulpitis, of whom 5\% requires treatment in one piece only and the rest between 2 or more dental pieces. The 39\% of the total sample presented cavities. There is no significant difference in the frequency of need for endodontic treatment or in the prevalence of cavities, between both sexes $\left(X^{2} C p>0.05\right)$
\end{abstract}

Key words: endodontics, prevalence, dental caries, pulpitis. 


\section{INTRODUCCIÓN}

En la actualidad a pesar de las mejoras que se han ido realizando en el área de salud oral en los distintos países de ingresos altos, la caries dental sigue siendo un importante problema de salud pública mundial, ${ }^{4}$ sobre todo países de ingresos bajos en donde los tratamientos de caries dental en los niños es prácticamente inexistente y muy limitado. ${ }^{7}$

La caries dental se ha convertido en un problema de salud pública afectando al $90 \%$ de la población mundial, derivando un gasto económico muy grande para los estados y/o para las personas, ${ }^{1}$ según cifras oficiales en Ecuador el Índice CPOD a los 12 años de edad es en promedio de $2.95 .^{2}$

En el 2007, la Organización Mundial de la Salud (OMS) publico el aumento en el número de enfermedades orales a nivel de todo el mundo y acentuó la necesidad de intensificar la recopilación de datos sobre la prevalencia de caries en los niños y sus consecuencias. ${ }^{8}$ En vista a los resultados obtenidos sobre la epidemia mundial de caries no tratadas en niños, existe una urgente necesidad de establecer un sistema de puntuación que evalúa y cuantifica los distintos estadios avanzados de caries.

Debido a la pobreza de datos emitidos acerca de un estudio epidemiológico de la prevalencia de caries dental no tratadas con compromiso pulpar o sepsis sobre un grupo de escolares, hemos visto necesario realizar nuestro primer estudio epidemiológico en la ciudad de Cuenca - Ecuador, además, los escasos datos disponibles no son fácilmente comparables debido a los diferentes sistemas de puntuación utilizados. ${ }^{9}$

El diagnóstico epidemiológico es una herramienta que permite conocer las características de distribución del proceso salud enfermedad. Permitiendo la observación, descripción, análisis y programación local para evitar la carga de enfermedades. $^{3}$

El presente estudio se logró mediante la colaboración binacional de dos universidades latinoamericanas.

\section{METODOLOGÍA}

Estudio transversal de evaluación clínica bucal en 130 escolares de 12 años de edad, de la comunidad urbana en Yanuncay Cuenca-Ecuador, esta muestra se calculó mediante fórmula:

$$
n=\frac{\left(Z^{2} \cdot p \cdot q \cdot N\right)}{Z^{2} \cdot p \cdot q+E^{2}(N-1)} ;
$$

en base a una población de 1398 escolares, con 0,05 de error y $95 \%$ de confiabilidad. Fueron incluidos pacientes con dentición permanente, fueron excluidos los que presentaron enfermedades sistémicas y malformaciones bucales, se utilizaron los criterios del índice P.U.F.A para la necesidad de tratamiento endodóntico, teniendo cuatro categorías:

P: PULPITIS $^{4}$, se registra en el momento que la cámara de la pulpa es visible o cuando las estructuras del diente han sido destruidas por el proceso de caries (Fig.1)

\begin{tabular}{lcccc}
\hline NEC. TRAT. & \multicolumn{2}{c}{ SEXO } & \multicolumn{2}{c}{ Total general } \\
ENDO. & FEMENINO & MASCULINO & $n$ & $\%$ \\
\hline ENFERMO & 5 & 4 & 9 & $7 \%$ \\
SANO & 60 & 61 & 121 & $93 \%$ \\
Total general & 65 & 65 & 130 & $100 \%$ \\
\hline & \multicolumn{3}{c}{ Chi cuadrado $p=0.73$}
\end{tabular}

Tabla 1. Necesidad de tratamiento endodóntico.

\begin{tabular}{ccc}
\hline $\begin{array}{c}\text { Número de piezas con } \\
\text { nec. tratamiento }\end{array}$ & Total general \\
\hline 0 & 121 & $\%$ \\
\hline 1 & 7 & $53 \%$ \\
2 & 1 & $1 \%$ \\
3 & 1 & $1 \%$ \\
Total general & 130 & $100 \%$ \\
\hline
\end{tabular}

Tabla 2. Piezas con necesidad de tratamiento

U: ÚLCERA , debido al trauma de afilados trozos de diente que han provocado ulceración traumática de los tejidos blandos circundantes, por ejemplo, la lengua o la mucosa bucal. $^{4}$ (Fig. 2)

F: FÍSTULA , cuando se libera pus debido a que el diente tiene compromiso pulpar. ${ }^{4}$ (Fig.3).

A: ABSCESOS, cuando un pus que contiene la inflamación relacionada con un diente con compromiso pulpar. ${ }^{4}$ (Fig.4) se usaron y los criterios epidemiológicos de la OMS para la prevalencia de caries. ${ }^{1}$

Los observadores fueron calibrados por un especialista (Kappa $>80 \%$ ), los padres firmaron el consentimiento y los escolares dieron el asentimiento informado. Todos los exámenes bucales fueron desarrollados con luz natural y espejo bucal, previos al consumo de alimentos por estudiantes de la UCACUE y el análisis estadístico fue realizado por los integrantes de la UANCV de Perú.

\section{RESULTADOS}

La muestra presentó $50 \%$ de personas de cada sexo; solo $61 \%$ fueron escolares sanos (Gráf.1) además $7 \%$ presentaron necesidad de tratamiento endodóntico, principalmente por pulpitis (Tabla 1 ), de los cuales el $5 \%$ requiere tratamiento en una pieza y el $95 \%$ entre 2 o más piezas dentales (Tabla 2). El 39\% del total de la muestra presentó caries. No existe diferencia significativa en la frecuencia de necesidad de tratamiento endodóntico ni en la prevalencia de caries, entre ambos sexos $X^{2}(p>0,05)$ (Tabla 3). 


\begin{tabular}{lcccc}
\hline & \multicolumn{2}{c}{ SEXO } & \multicolumn{2}{c}{ Total general } \\
\multicolumn{1}{c}{ CARIES } & FEMENINO & MASCULINO & $n$ & $\%$ \\
\hline ENFERMO & 27 & 24 & 51 & $39 \%$ \\
SANO & 38 & 41 & 79 & $61 \%$ \\
Total general & 65 & 65 & 130 & $100 \%$ \\
\hline
\end{tabular}

Tabla 3. Prevalencia de caries.

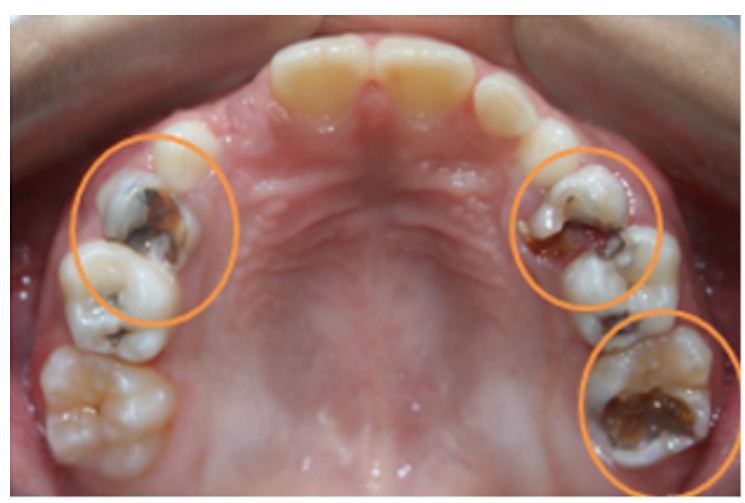

Fig. 1. Piezas dentales con la cámara pulpar visible debido a una destrucción de la estructura dental por caries.

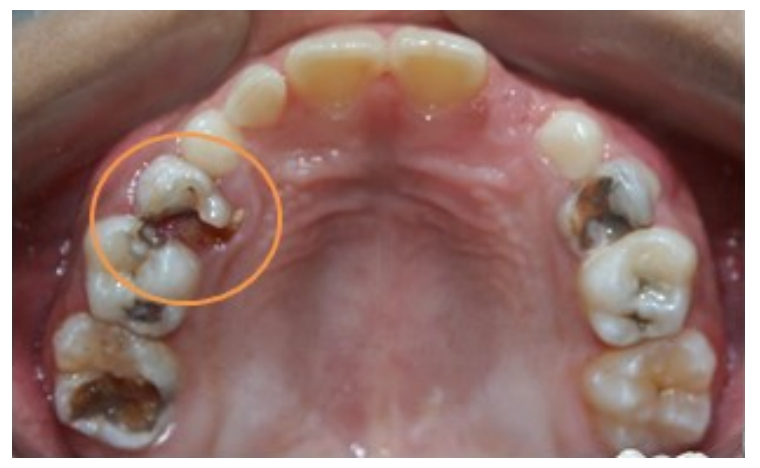

Fig. 2. Presencia de lesión en los tejidos blandos debido a estructuras dentales punzantes.

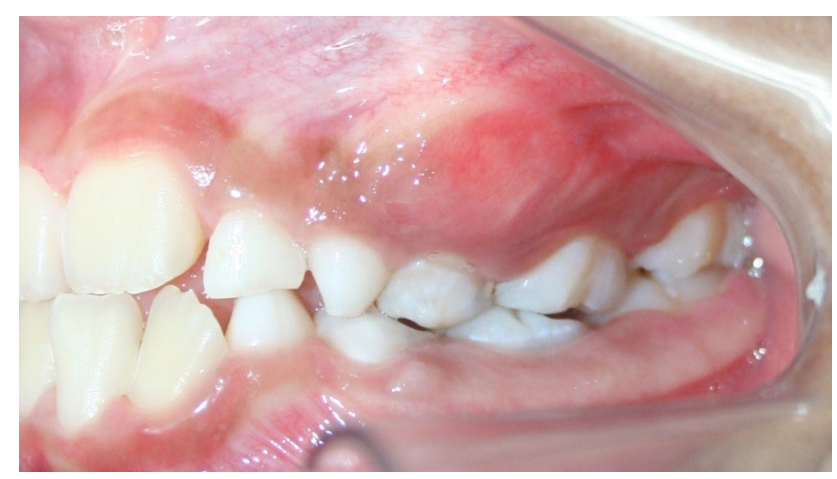

Fig. 3. Presencia de fístula.

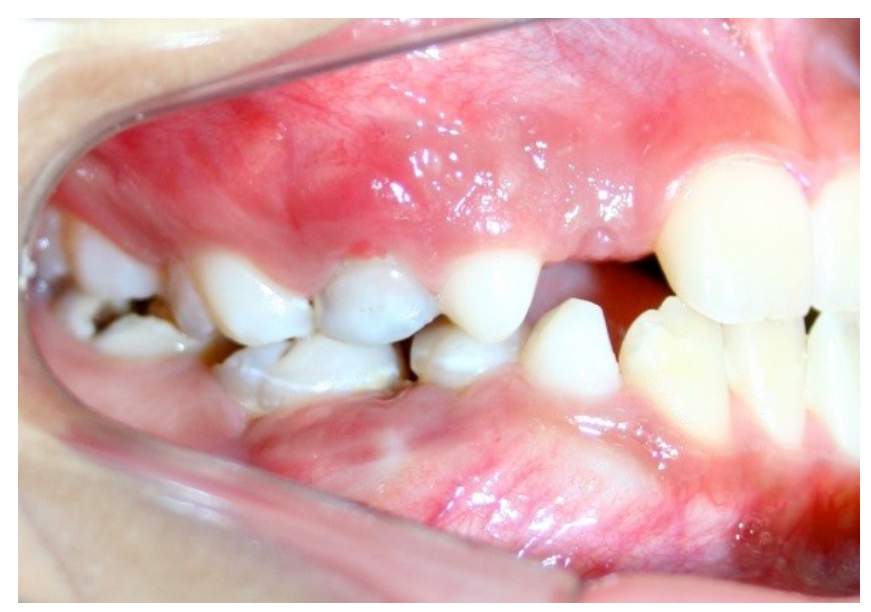

Fig. 4. Presencia de absceso dental y secreción de sustancias purulentas.

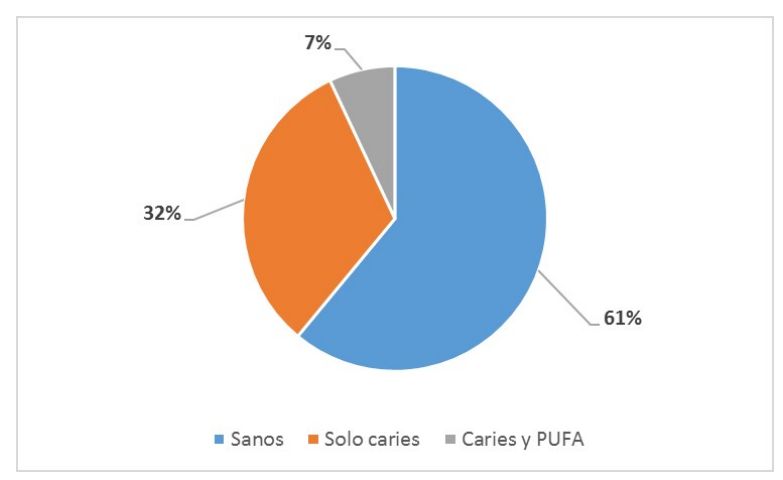

Grf. 1. Caries y necesidad de tratamiento endodóntico.

\section{DISCUSIÓN}

Existe elevada prevalencia de caries dental en niños de 12 años de edad, siendo piezas permanentes finalizando su erupción. Este tipo de condiciones orales no son solo un problema en nuestro país, sino que de la misma forma afecta a diferentes partes de Latino América como es el caso de la ciudad de Paranoá, Brazil, cuyo estudio del índice PUFA en escolares fue de $23,7 \%,{ }^{5}$ un valor más elevado con los hallazgos de nuestro estudio, puesto que se realizó en escolares de 6-7 años y ellos muestran evidentemente más índice PUFA que los escolares de 12 años de edad, por la dentición mixta.

Un estudio realizado en la ciudad de Ventanilla, Perú respecto a las consecuencias clínicas de caries dental no tratada, la frecuencia de PUFA fue de $1.29 \%$ de las piezas evaluadas, el cual representa un valor superior al obtenido en nuestra investigación, dado que se hizo en dientes permanentes y deciduos. ${ }^{3}$ Un estudio en Alemania, reportó una prevalencia de infecciones odontogénicas de $4,4 \%$, un 
valor que difiere al de nuestro país, debido a la falta de un plan escolar de prevención. ${ }^{6}$ Se estima que con cada año, la probabilidad de necesitar un tratamiento endodóntico aumenta en $1,16 \%$.

\section{CONCLUSIÓN}

Este levantamiento de datos revela la existencia de una prevalencia de caries dental alta a pesar de la corta edad de los escolares y que existe necesidad de tratamiento endodóntico a los 12 años de edad.

\section{Referencias}

1 Petersen PE. Continuous improvement of oral health in the 21st century. The World Oral Health Report. 2003: p. 4.

2 Reinoso-Vintimilla N. Villavicencio-Caparó E. Caries dental en escolares de 12 años de la parroquía Sayausí (Cuenca). Odontología Activa ucacue. 2015; p. 34

3 Pachas-Barrionuevo F, García-Zavaleta C, Carrasco-Loyola M, Manrique-Chávez J, Orejuela-Ramírez F, CórdovaSotomayor D, et al. Facultad de Estomatología upch master challenge. [Online].; 2015. Documento disponible

4 Monse B. pufa-An index of clinical. Community Dent Oral Epidemiol. 2010. Documento disponible

5 Sé M. Prevalência de cárie em escolares do Paranoá. Repositorio Institucional Universidad de Brasilia. 2011;: p. 7. Documento disponible

6 Grund K. Clinical consequences of untreated dental caries in German 5- and 8-year-olds. Bio Med Central. 2015 noviembre. Documento disponible

7 Pitts NB, Boyles J, Nugent ZJ, Thomas N, Pine CM. The dental caries experience of 5-year-old children in Great Britain (2005/6) . Surveys co-ordinated by the 81 Clinical consequences of untreated dental caries British Association for the Study of Community Dentistry. Community Dent Health 2007;24:59-63.

8 World Health Organization. Oral Health: action plan for the promotion and integrated disease prevention. Sixtieth World Health Assembly. WHA60.17. Agenda item 12.9.2007.

9 Pine C, Harris V, Burnside G, Merrett M. An investigation of the relationship between untreated decayed teeth and sepsis in 5-year-old children. British Dent J 2006;200:45-7.

Recibido: 5 de Abril de 2016

Aceptado: 15 de Abril de 2016 